\begin{tabular}{|c|c|}
\hline $\begin{array}{l}\text { REVISTA } \\
\text { E.ŨACIÓN }\end{array}$ & $\begin{array}{l}\text { Revista Educación } \\
\text { ISSN: 0379-7082 } \\
\text { ISSN: } 2215-2644 \\
\text { revedu@gmail.com } \\
\text { Universidad de Costa Rica } \\
\text { Costa Rica }\end{array}$ \\
\hline
\end{tabular}

\title{
Calidad en la educación superior en línea: un análisis teórico
}

García Soto, Gladys Yvone; García López, Ramona Imelda; Lozano Rodríguez, Armando

Calidad en la educación superior en línea: un análisis teórico

Revista Educación, vol. 44, núm. 2, 2020

Universidad de Costa Rica, Costa Rica

Disponible en: http://www.redalyc.org/articulo.oa?id=44062184005

DOI: https://doi.org/10.15517/revedu.v44i2.39714

Esta obra está bajo una Licencia Creative Commons Atribución-NoComercial-SinDerivar 3.0 Internacional. 
Revisiones bibliográficas

\title{
Calidad en la educación superior en línea: un análisis teórico
}

\author{
Quality in Online Higher Education: A Theoretical Analysis \\ Gladys Yvone García Soto \\ DOI: https://doi.org/10.15517/revedu.v44i2.39714 \\ Instituto Tecnológico de Sonora, México \\ Redalyc: http://www.redalyc.org/articulo.oa?id=44062184005
}

maestragladysgarcia@gmail.com

iD http://orcid.org/0000-0002-9447-3073

Ramona Imelda García López

Instituto Tecnológico de Sonora, México

igarcia@itson.edu.mx

iD http://orcid.org/0000-0003-0091-3427

Armando Lozano Rodríguez

Instituto Tecnológico de Sonora, México

armando.lozano@itson.edu.mx

http://orcid.org/0000-0002-7013-4210

Recepción: 20 Diciembre 2019

Aprobación: 10 Abril 2020

\section{RESUMEN:}

El incremento de la oferta educativa en modalidades no convencionales genera la necesidad de evidenciar que los modelos educativos son de calidad. Mediante un análisis documental y comparativo el presente trabajo tiene el objetivo de mostrar los principales estándares nacionales e internacionales tomados en cuenta por organismos evaluadores de la calidad educativa, así como la complejidad que ha implicado dicho proceso en el sistema educativo mexicano y a nivel internacional. Para el análisis se desarrolló una matriz de criterios y categorías establecidos en los marcos de evaluación de organismos evaluadores como CIEES, Conacyt, CALED y OLC, con el fin de compararlos, e identificar la ponderación o relevancia que le otorgan para determinar la calidad de los programas. Como resultado de la comparación se identificaron ocho dimensiones en torno a la evaluación de calidad: modelo educativo, resultados del modelo, estudiantes, personal docente, infraestructura, servicios y procesos administrativos, responsabilidad social de la institución y vinculación. A partir de los hallazgos, se recomienda la estandarización de los aspectos a evaluar por parte de las instituciones evaluadoras, mediante la unificación del número de categorías o criterios; así como también se considera necesario especificar modelos evaluadores adecuados para la educación en modalidades no convencionales.

Palabras Clave: Calidad educativa, Educación a distancia, Evaluación, Educación superior.

\section{Abstract:}

The rise of non-conventional educational modalities has triggered the need to verify the quality of such educational models. This documentary and comparative analysis demonstrates current national and international standards upheld by quality assurance bodies and the complex process of enforcing these standards within Mexico's educational system and at international levels. A rubric of criteria and categories used by educational assessment bodies such as CIEES (Interinstitutional Committee for the Evaluation of Higher Education in Mexico), Conacyt (National Council of Science and Technology), CALED (Latin America and Caribbean Institute for Quality in Distance Higher Education) and OLC (Online Learning Consortium) was developed for comparative purposes and to identify how these bodies weigh or determine the relevance of each attribute when determining program quality. As a result of this comparison, eight dimensions associated with educational evaluations were identified: educational models, results of the model, students, faculty, infrastructure services and administrative procedures, institutional social responsibility and linkages. Based on the findings, recommendations are made to standardize aspects evaluated by the assessment bodies, such as consolidating the number of categories or criteria and, assign appropriate assessment models for nonconventional education modalities.

KEYWORDs: Educational Quality, Distance Learning, Evaluation, Assessment, Higher Education. 


\section{INTRODUCCIÓN}

La educación superior es considerada como base para un buen desarrollo socioeconómico debido a la formación de capital humano que esta permite. Sin embargo, sigue presentándose un problema de desigualdad de ingreso a las instituciones educativas. Por ello, la modalidad de educación a distancia (EaD) ha representado una de las estrategias implementadas con el propósito de ampliar la cobertura y los procesos formativos. Para conocer el impacto y resultados de programas e instituciones que ofertan modalidades educativas no convencionales se utilizan mecanismos de evaluación de la calidad en los sistemas formadores, como redes de colaboración que le den impulso y sistemas para su evaluación (García, Ruiz, Quintanal, García y García, 2009).

Sin embargo, se señala que en el contexto de la educación a distancia se requiere de una actualización en dichos procesos debido a que los instrumentos utilizados son considerados imprecisos y por tanto no han evidenciado los resultados de estas nuevas modalidades educativas (Morocho y Rama, 2015). Para Rama (2015) un sistema de evaluación educativa debe contemplar todas sus dimensiones, áreas y variables del fenómeno universitario, desde su gestión e implementación hasta sus resultados transformados en aprendizajes, analizándolo desde su complejidad. Para ello, el autor considera que se requiere de sistemas de evaluación que sistematicen la gran cantidad de datos obtenidos en el proceso.

Analizar por tanto la educación superior en cualquiera de sus modalidades exige evaluaciones las cuales permitan dimensionar y cualificar todos aquellos aspectos que muestren a la institución en su realidad; de esta forma se requiere especificar cuáles deberían ser los aspectos reales y más importantes de la educación a distancia; es decir, los aprendizajes significativos (Rama, 2015).

Demostrar la calidad de la enseñanza en modalidades no escolarizadas representa un gran reto para las instituciones de educación superior (IES) en México. Esto debido a las complejidades que se muestran en las metodologías de evaluación de los organismos nacionales, tales como la falta de mecanismos de evaluación diseñados específicamente para la modalidad, problema que tiene su origen en la falta de consistencia en las normas y leyes que dan forma a la educación en dicha modalidad (Cervantes, Bañuelos, Chávez y Rocha, 2015).

Sin embargo, no solo en México se han presentado complejidades en los procesos de evaluación, acreditación o certificación de la educación a distancia, sino también en Latinoamérica, en donde se evidencia la falta de unificación de criterios, estándares o lineamientos en las metodologías de evaluación implementadas por parte de los organismos encargados de efectuar dichos procesos (Rama, 2015).

Por ello, en el presente documento se exponen los distintos estándares de calidad evaluados por organismos acreditadores con el objetivo de analizar las distintas categorías de evaluación de calidad en la educación superior a distancia a nivel internacional, para ofrecer una descripción de los insumos que son evaluados y las terminologías utilizadas por cada uno de ellos; así mismo, se pretende analizar los trabajos encaminados a las mejoras en los procesos de evaluación de calidad en países de Latinoamérica, con la intención de brindar a las IES un mapeo en torno a los sistemas evaluativos en el plano nacional e internacional.

\section{Inicios de LAS MODALIDADES No CONVENCIONALES EN MÉXico}

En la educación superior mexicana, la Secretaría de Educación Pública [SEP] reconoce tres modalidades educativas: escolarizada, no escolarizada y mixta (Diario Oficial de la Federación, 1998). La primera de ellas es definida como aquellos servicios educativos que se ofertan en espacios físicos en donde estudiantes reciben su formación académica. La no escolarizada es donde el alumnado no asiste a sus estudios en instalaciones escolares; asume el significado de educación en línea, virtual, a distancia, on line, entre otras denominaciones. Y en combinación de las anteriores, se sitúa la educación mixta. 
La modalidad educativa no escolarizada en México tuvo su aparición en los años cuarenta con la intención de hacer llegar los aprendizajes a personas que por distintos motivos no podían presentarse en los planteles educativos. Así mismo, en el año 1966 se pone en marcha la enseñanza mediante la Telesecundaria con el objetivo de ofrecer educación media en aquellas poblaciones con problemas de acceso (Navarrete y Manzanilla, 2017).

Adicionalmente, con los avances tecnológicos que se han presentado en las últimas décadas, la modalidad a distancia tuvo un gran auge y las IES la han implementado como un mecanismo que les permite la masificación de su matrícula (Mena, Rodríguez y Diez, 2005). Es por ello que, desde la llegada de las Tecnologías de la Información y Comunicación, dicha modalidad (TIC) se ha incrementado exponencialmente en México (SEP, 2018).

\section{CALIDAD EN LA EDUCACión A Distancia}

El impacto de la educación superior puede ser medido mediante la calidad educativa (García et al., 2009), y esta se define como una característica de la educación representada mediante la satisfacción de sus clientes (estudiantes) (Marciniak, 2016). Para Arias (2015), calidad es un proceso complejo, y más aún cuando se refiere a la educación a distancia debido sus cambios constantes.

Una definición global de calidad es la ofrecida por la Real Academia Española (RAE) describiéndola como un atributo o propiedad que caracteriza o determina el valor de un objeto, proceso o producto (Real Academia Española [RAE], 2018). Sin embargo, para entornos educativos, la calidad es representada como la eficiencia en los procesos y la congruencia de estos con sus resultados; los cuales le permitan cumplir con las expectativas de quienes son considerados clientes de la educación (Fernández, 2004).

Desde la perspectiva de Alcántara (2007), la calidad educativa, específicamente en el ámbito de educación superior, no es un concepto que pueda ser explicado por sí solo, este requiere ser analizado desde diversos componentes o dimensiones; convirtiéndose en uno de los principales atributos de la educación que contempla desde sus procesos, instituciones y establecimientos.

La Unesco, en la Conferencia Internacional sobre la Educación Superior celebrada en 1998, postula que la calidad debía comprender todas las actividades y funciones contenidas en el marco de la educación; desde la enseñanza, evaluación, programas de curso y estudio, profesores, estudiantes, hasta los servicios ofrecidos, infraestructura y equipo (Alcántara, 2007).

Es así como la Unesco (2016) define calidad educativa desde cinco dimensiones:

a) Relevancia: referida a las finalidades que le son asignadas a la educación, tanto en los contextos político y social. Una educación en donde su currículo se encuentre en beneficio de la conexión entre los aprendizajes, el contexto y el estudiantado.

b) Pertinencia: donde se señala que la educación deberá ser significativa para cada individuo, permitiendo la apropiación de los contenidos y la enseñanza en sí. Es decir, el centro de todo proceso educativo deberá ser el estudiantado, y los procesos educativos deberán enfocarse en él.

c) Equidad: igualdad de oportunidad de acceso a la educación de todas las personas. Para la Unesco la educación solo es de calidad cuando es para todos.

d) Eficacia: esta dimensión será entendida conforme al logro de los objetivos que son propuestos en los sistemas educativos, o en qué medida se garantizan los principios establecidos mediante la operacionalización de metas.

e) Eficiencia: la medida en que la educación maneja los recursos que solventan a los sistemas educativos; es decir, el resultado de la administración y la transparencia de dicho proceso.

Por su parte, la Organización para la Cooperación y el Desarrollo Económico (OCDE) (Marciniak, 2016) define la calidad educativa como ese factor que brinda a cada estudiante seguridad en cuanto a la adquisición 
de aprendizajes, habilidades y competencias que le permitan un desarrollo profesional en su etapa de adultez, así como un elemento que se centra en la búsqueda constante de la excelencia y el mejoramiento del quehacer educativo. Sin embargo, la autora considera que dicha definición para la educación en modalidades no convencionales resulta compleja, asociada a los procesos y productos del aprendizaje. Por otra parte, Rama (2016), la conceptualiza como una expresión de la interacción de las competencias adquiridas, asociadas a las respectivas pedagogías, y en función de las demandas sociales. Desde otra perspectiva, para Marciniak y Gairín (2017) la calidad en lo virtual se define como el grado de satisfacción en el cual el alumnado se encuentra con respecto de la formación que le brinda la institución.

\section{EVALUACIÓN DE LA CALIDAD EN LA EDUCACIÓN VIRTUAL Y A DISTANCIA}

Hasta este punto no ha sido posible llegar a una postura unificada sobre la calidad en la educación; así como abordar su estudio desde procesos de evaluación en entornos educativos no escolarizados (García et al., 2009; Marciniak, 2016). Es por ello que, algunas organizaciones y las IES, se han preocupado por evidenciar la evaluación de la calidad en los modelos educativos. Desde una postura de análisis, se considera que debido a la situación cambiante de la modalidad virtual se requiere de nuevos mecanismos o la innovación de los procesos ya existentes en cuanto a criterios que deberían ser considerados para su evaluación. Tal como se ha mencionado, la evaluación de la calidad en la $\mathrm{EaD}$ es un problema; por tanto, se considera relevante conocer las experiencias respecto a procesos de evaluación de la calidad en los sistemas de educación a distancia (Morocho y Rama, 2015).

Sin duda, conocer el proceder de las evaluaciones de calidad en torno a la educación a distancia depende de identificar aquellas organizaciones dedicadas a evaluar y acreditar a las IES; por ello, se presenta a continuación un marco general de aquellas que han sido consideradas factores clave en el desarrollo de evaluaciones en las modalidades no escolarizadas.

A manera de recuento histórico sobre el surgimiento de los procesos acreditación, Domínguez (2016) expone que en Estados Unidos las evaluaciones dieron inicio en el siglo XIX en universidades privadas, mientras que en Colombia se desarrollan desde el año 1984, y en 1992 se crea el Sistema Nacional de acreditación en dicho país. En Brasil se cuenta con el Programa de Evaluación Institucional de las Universidades Brasileñas, creado en 1993; sin embargo, sus procesos de acreditación inician desde 1977 a nivel de posgrados. Así mismo, con el propósito de analizar los procesos de acreditación de la modalidad virtual y a distancia en América Latina, el autor expone que el surgimiento de las evaluaciones se ha visto favorecido por las reformas del Banco Mundial, que han brindado su apoyo y direccionado investigaciones a dichos procesos debido al incremento de la matrícula de las IES.

Para el caso de Ecuador, se cuenta con el Consejo Nacional de Evaluación y Acreditación de la Educación Superior del Ecuador (CONEA). Así mismo, la calidad educativa en España se rige bajo la Ley Orgánica de Universidades (LOU) (Domínguez, 2016). En México se efectúan evaluaciones desde los años setenta; en este país el Consejo para la Acreditación de la Educación Superior (COPAES) es el organismo reconocido por la SEP para el otorgamiento oficial de acreditación en calidad.

En España, la Agencia Nacional de Evaluación de la Calidad y Acreditación (ANECA) es el organismo de evaluación de la calidad de la educación superior responsable de implementar procesos de evaluación, certificación y acreditación, del sistema universitario español con el propósito de su mejora continua y adaptación al Espacio Europeo de Educación Superior (EEES). Para el logro de sus objetivos, dicho organismo desarrolla evaluaciones a programas de Grado, Máster y Doctorado, a organismos y procesos académicos institucionales, al profesorado, así como a la producción de investigaciones de los profesores mediante la Comisión Nacional de Evaluación de la Actividad Investigadora (CNEAI) (ANECA, 2018a). 
La ANECA organiza sus actividades de evaluación, certificación y acreditación mediante varios procesos: programas de evaluación al profesorado, programas de evaluación de títulos, y programas de evaluación institucional; en estos últimos se cuenta con tres procedimientos:

- AUDIT: orienta a los centros universitarios en el diseño de sistemas de garantía interna de calidad.

- ACREDITACIÓN INSTITUCIONAL: evalúa las solicitudes de acreditación institucional de los centros universitarios.

- DOCENTIA: Ayuda a las universidades a crear sistemas de evaluación de su profesorado (ANECA, 2018b).

Este último es el programa mediante el cual se ejecuta el proceso de acreditación institucional de los centros educativos, con un ámbito de actuación en universidades dependientes de comunidades autónomas cuya responsabilidad de la acreditación de títulos corresponda a ANECA, universidades establecidas en comunidades autónomas sin órgano de evaluación externa inscrito en el Registro Europeo de Agencias de Calidad (European Quality Assurance Register, EQAR) y la Universidad Nacional de Educación a Distancia.

Como resultado de una revisión bibliográfica y documental, Marciniak (2016) engloba la evaluación de la calidad en la educación virtual desde las siguientes categorías: a) justificación y objetivos del modelo, b) perfil de ingreso y egreso, c) contenido curricular, actividades, materiales y recursos didácticos, d) diseño instruccional, y e) evaluación de los aprendizajes. Concluye que los procesos de evaluación requieren innovación constante y esta deberá desarrollarse en las distintas fases, creación, desarrollo e implementación.

En una comparación de los estándares de calidad en convergencia Chile-España Domínguez (2016) categoriza los siguientes criterios:

- Perfil de egreso: presentado de manera clara y explícita, difundido de manera adecuada, actualizado, que se muestre consistencia entre el perfil, el plan de estudios y las estrategias pedagógicas, existencia de procedimientos para la actualización del perfil, y que considere opiniones de los actores externos que revisan dicho perfil.

- Estructura curricular: que se encuentre actualizada y flexible, existencia de prácticas profesionales, programas de clase actualizados, y no sobrecargar los planes de estudio.

- Ex estudiantes y empleadores: políticas de seguimiento de egresados, políticas operativas que aseguren la comunicación con ex estudiantes, conocimiento del puesto de su trabajo, y existencia de los mecanismos de consulta con empleadores y personas egresadas.

- Resultados: índices de aprobación, seguimiento de las trayectorias del alumnado, existencia de indicadores en cuanto a aspectos docentes, y tiempo de egreso satisfactorio.

- Práctica docente: métodos pedagógicos adecuados, seguimiento de procesos formativos, y mecanismos para la verificación del logro de las competencias.

Por su parte, el Comité para la Evaluación de la Educación Superior (CIEES) en México, delimita los ejes que se muestran en la Tabla 1 para llevar a cabo sus evaluaciones: 
TABLA 1

Ejes y categorías evaluados por CIEES

\begin{tabular}{|c|c|}
\hline Ejes & Categorias \\
\hline \multirow{2}{*}{$\begin{array}{l}\text { I. Fundamentos y condiciones de } \\
\text { operación }\end{array}$} & 1. Propósitos del Programa \\
\hline & $\begin{array}{l}\text { 2. Condiciones generales de operación del } \\
\text { programa }\end{array}$ \\
\hline \multirow[t]{2}{*}{ II. Currículo específico genérico } & 3. Modelo educativo y plan de estudios \\
\hline & 4. Actividades para la formación integral \\
\hline \multirow{4}{*}{$\begin{array}{l}\text { III. Tránsito de estudiantes por el } \\
\text { programa }\end{array}$} & 5. Proceso de ingreso \\
\hline & 6. Trayectoria escolar \\
\hline & 7. Egreso del programa \\
\hline & 8. Resultados de los estudiantes \\
\hline \multirow{4}{*}{$\begin{array}{l}\text { IV. Personal académico, } \\
\text { infraestructura y servicios }\end{array}$} & 9. Personal académico \\
\hline & 10. Infraestructura académica \\
\hline & 11. Infraestructura física \\
\hline & 12. Servicios de apoyo \\
\hline
\end{tabular}

Fuente: Proceso general para la evaluación de programas educativos de educación superior (CIEES, 2017).

Otro de los organismos que regulan la educación superior en el contexto mexicano es la Asociación Nacional de Universidades e Instituciones de Educación Superior (ANUIES), que establece estándares y criterios enmarcados al posgrado en colaboración con el Consejo Nacional de Ciencia y Tecnología (Conacyt), quien ofrece un marco de referencia para la evaluación de la calidad de programas educativos no escolarizados (Rocha, Maina, y Sangra, 2015). Los criterios de calidad evaluados y sus categorías se muestran en la Tabla 2.

TABLA 2

Criterios de análisis del Conacyt

\begin{tabular}{|c|c|}
\hline Categoría & Criterios \\
\hline \multirow{2}{*}{$\begin{array}{l}\text { Responsabilidad social de la } \\
\text { institución }\end{array}$} & Compromiso Institucional \\
\hline & $\begin{array}{l}\text { Sistema interno de aseguramiento de la } \\
\text { calidad }\end{array}$ \\
\hline \multirow{4}{*}{$\begin{array}{l}\text { Estructura y personal académico del } \\
\text { programa }\end{array}$} & Plan de estudios \\
\hline & Proceso de enseñanza-aprendizaje \\
\hline & Núcleo académico básico \\
\hline & $\begin{array}{l}\text { Líneas de generación y lo aplicación del } \\
\text { conocimiento }\end{array}$ \\
\hline \multirow[t]{2}{*}{ Estudiantes } & Ingreso \\
\hline & Seguimiento de la trayectoria académica \\
\hline \multirow[t]{3}{*}{ Infraestructura y servicios } & $\begin{array}{l}\text { Tecnologías para el aprendizaje y servicios } \\
\text { de administración a distancia }\end{array}$ \\
\hline & $\begin{array}{l}\text { Espacios, equipamiento, laboratorios y } \\
\text { talleres }\end{array}$ \\
\hline & Información y documentación \\
\hline \multirow[t]{5}{*}{ Resultados y vinculación } & $\begin{array}{l}\text { Trascendencia, cobertura y evolución del } \\
\text { programa }\end{array}$ \\
\hline & Efectividad del posgrado \\
\hline & Contribución al conocimiento \\
\hline & Vinculación \\
\hline & Financiamiento \\
\hline
\end{tabular}

Fuente: Marco de referencia para la evaluación y seguimiento de programas de posgrado en modalidad no escolarizada (Conacyt, 2016). 
Así mismo, el Instituto Latinoamericano y del Caribe de Calidad en Educación Superior a Distancia (CALED) presenta las dimensiones de evaluación que se muestran en la Tabla 3, contenidas en la Tarjeta de puntuación (SCCQAP), evaluación de programas de pregrado en líne.(CALED y OLC, 2015): 
TABLA 3

Categorías e indicadores de la evaluación CALED/OLC

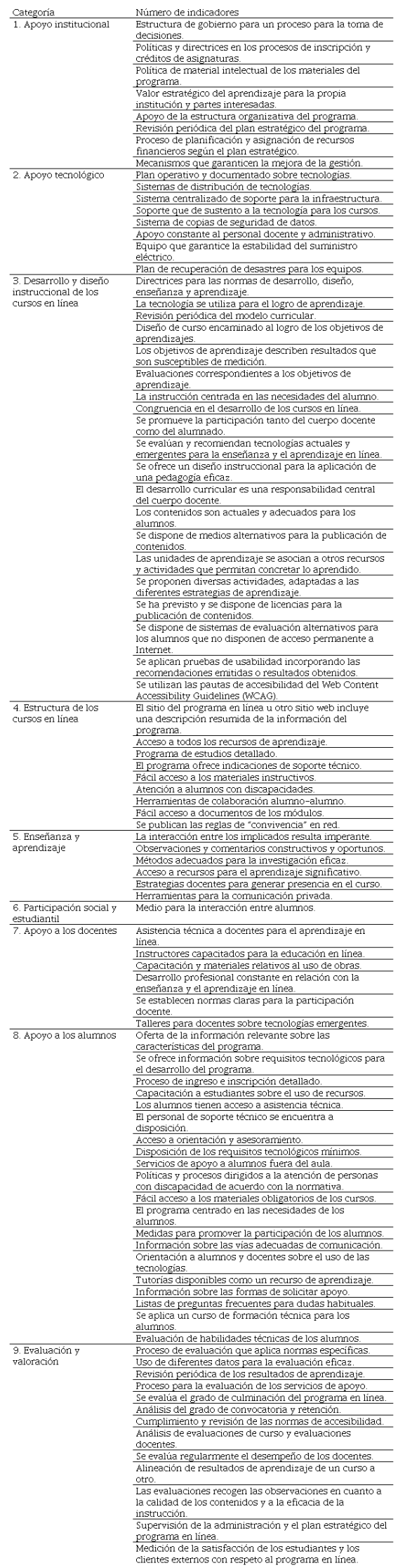


Fuente: Tarjeta de puntuación (SCCQAP), evaluación de programas de pregrado en línea (CALED y OLC, 2015).

Así mismo, el CALED ofrece a las IES su guía para la autoevaluación para programas de pregrado a distancia (CALED, 2010) en donde permite a las universidades evaluarse mediante 9 criterios, 30 subcriterios, 79 objetivos, 149 estándares y 333 indicadores (ver Tabla 4). 
TABLA 4

Criterios de evaluación CALED, guía para la autoevaluación

\begin{tabular}{|c|c|}
\hline Criterios & Objetivos \\
\hline 1. Liderazgo y estilos de gestión & $\begin{array}{l}\text { 1.1. Cultura de excelencia del programa, } \\
\text { en el marco de la Política y Estrategia de } \\
\text { la Institución con la que debe estar } \\
\text { alineada. 1.2. Trabajo activo con } \\
\text { personas de la Institución o ajenas a ella, } \\
\text { para promover y desarrollar los intereses } \\
\text { y satisfacer las expectativas actuales y } \\
\text { futuras de los agentes implicados en la } \\
\text { organización del programa. } 1.3 \text {. } \\
\text { Estructura organizacional desarrollada } \\
\text { para sustentar la eficaz y eficiente } \\
\text { aplicación de la Política y la Estrategia } \\
\text { relacionada con el programa. 1.4. Gestión } \\
\text { y mejora de procesos del programa } \\
\text { acordes a la gestión y mejora continua de } \\
\text { la Institución }\end{array}$ \\
\hline 2. Política y estrategia & $\begin{array}{l}\text { 2.1. Los objetivos del programa están } \\
\text { basados en las necesidades y } \\
\text { expectativas actuales y futuras de los } \\
\text { agentes implicados. } 2.2 \text {. Los objetivos del } \\
\text { programa están basados en información } \\
\text { pertinente y completa que proporciona } \\
\text { un marco de referencia. 2.3. El desarrollo } \\
\text { de la planificación estratégica del } \\
\text { programa se revisa para su continua } \\
\text { adecuación, se actualiza y mejora } \\
\text { periódicamente. 2.4. La planificación } \\
\text { estratégica del programa es comunicada } \\
\text { y entendida dentro de la Institución y en } \\
\text { los agentes implicados en su desarrollo. }\end{array}$ \\
\hline 3. Desarrollo de las personas & $\begin{array}{l}\text { 3.1. Los responsables del programa } \\
\text { planifican y mejoran la gestión de su } \\
\text { personal. 3.2. Los responsables del } \\
\text { programa identifican, mantienen y } \\
\text { desarrollan la experiencia y capacidades } \\
\text { de las personas. } 3.3 \text {. Los responsables } \\
\text { del programa promueven la implicación y } \\
\text { participación de todo su personal en la } \\
\text { mejora continua. 3.4. Los responsables } \\
\text { del programa consiguen una } \\
\text { comunicación efectiva ascendente, } \\
\text { descendente y lateral. } 3.5 \text {. Los } \\
\text { responsables del programa reconocen, } \\
\text { atienden y recompensan al personal } \\
\text { involucrado en el mismo. }\end{array}$ \\
\hline 4. Recursos y alianzas & $\begin{array}{l}\text { 4.1. Gestión de los recursos económicos y } \\
\text { financieros del programa. } 4.2 \text {. Gestión de } \\
\text { los recursos de Información. 4.3. Gestión } \\
\text { de los recursos externos y alianzas. } 4.4 \text {. } \\
\text { Gestión de infraestructura física y } \\
\text { tecnológica. }\end{array}$ \\
\hline 5. Destinatarios y procesos educativos & $\begin{array}{l}\text { 5.1. Identificación de los alumnos. } 5.2 \text {. } \\
\text { Identificación de los procesos educativos } \\
\text { para el desarrollo del programa. } 5.3 \text {. } \\
\text { Evaluación y mejora del programa. }\end{array}$ \\
\hline $\begin{array}{l}\text { 6. Resultados de destinatarios y procesos } \\
\text { educativos }\end{array}$ & $\begin{array}{l}\text { 6.1. Medidas del Grado de Satisfacción de } \\
\text { los Destinatarios y Procesos Educativos. } \\
\text { 6.2. Medidas del Desempeño y } \\
\text { Rendimiento. }\end{array}$ \\
\hline $\begin{array}{l}\text { 7. Resultados del desarrollo de las } \\
\text { personas }\end{array}$ & $\begin{array}{l}\text { 7.1. Medidas de la Percepción del } \\
\text { Personal. } 7.2 \text {. Medidas del Desempeño y } \\
\text { Rendimiento. }\end{array}$ \\
\hline 8. Resultados de sociedad & $\begin{array}{l}\text { 8.1. Medidas de la Percepción de la } \\
\text { Sociedad. 8.2. Medidas del Desempeño y } \\
\text { Rendimiento. }\end{array}$ \\
\hline 9. Resultados globales & $\begin{array}{l}\text { 9.1. Resultados obtenidos por la } \\
\text { Institución. 9.2. Resultados obtenidos } \\
\text { por las Personas implicadas en el } \\
\text { programa. 9.3. Resultados obtenidos por } \\
\text { los Alumnos y otros Clientes Externos. } \\
\text { 9.4. Resultados obtenidos por la } \\
\text { Sociedad. }\end{array}$ \\
\hline
\end{tabular}


Fuente: Guía para la autoevaluación para programas de pregrado a distancia (CALED, 2010).

\section{Metodología}

Se elaboró un análisis documental de los principales marcos de referencia con respecto a la acreditación de programas educativos en modalidades no convencionales. Los organismos acreditadores contemplados fueron CIEES, Conacyt, CALED, y OLC. El criterio de selección de los organismos certificadores y por consecuencia de los marcos de referencia fue la disponibilidad con la que las instituciones contaron para el acceso a sus documentos de evaluación, el cual se ejecutó mediante la información con la que dichas organizaciones cuentan en su sitio web.

Una vez analizados dichos documentos, se estructuró una base de datos con los principales criterios y categorías de evaluación; lo cual permitió la generación de una lista de verificación. Posterior a la generación de dicha lista de códigos se seleccionaron los criterios que conformaron los resultados con base en su relevancia, ponderación e incidencia en los documentos analizados.

Se analizaron y compararon un total de 150 criterios de evaluación distribuidos en 27 dimensiones contenidas en los cuatro marcos de referencias (ver Tabla 5).

TABLA 5

Dimensiones de evaluación por organismo acreditador

\begin{tabular}{llll} 
Conacyt & CIEES & CALED /OLC & CALED \\
\hline 1. Responsabilidad social de la institución & 1. Fundamentos y condiciones de operación & 1. Apoyo institucional & 1. Liderazgo y estilos de gestión \\
\hline 2. Estructura y personal académico del programa & 2. Currículo específico genérico & 2. Apoyo tecnológico & 2. Política y estrategia \\
\cline { 2 - 3 } & 3. Tránsito de los estudiantes por el programa & 3. Desarrollo y diseño instruccional de los cursos en línea & 3. Desarrollo de las personas \\
\hline 3. Estudiantes & 4. Personal académico, infraestructura y servicios & 4. Enseñanza y aprendizaje & 4. Recursos y alianzas \\
\hline 4. Infraestructura y servicios & 5. Estructura de los cursos en línea & 5. Destinatarios y procesos educativos \\
\hline 5. Resultados y vinculación & 6. Participación social y estudiantil & 6. Resultados de destinatarios y procesos educativos \\
\hline & 7. Apoyo a los docentes & 7. Resultados del desarrollo de las personas \\
\hline & 8. Apoyo a los alumnos & 8. Resultados de sociedad & 9. Resultados globales
\end{tabular}

Fuente: Elaboración propia.

\section{Resultados}

Como resultado del análisis documental de los marcos de referencia e investigaciones enfocadas en la calidad, mediante una lista de verificación se han delimitado ocho dimensiones o categorías de evaluación establecidas por algunos organismos acreditadores. Lo anterior, permitió determinar los elementos comunes que comparten dichos organismos; estos se presentan en la Tabla 6. 
TABLA 6

Análisis de criterios de calidad

Dimensión

I. Modelo educativo.

II. Resultados del modelo educativo.

III. Alumnos.

IV. Personal docente

Infraestructura

VI. Servicios y pro

VII. Responsabilidad social de la institución

\begin{tabular}{|c|c|}
\hline & $\begin{array}{l}\text { C27. Mecanismos para la movilidad y el intercambio } \\
\text { escolar. }\end{array}$ \\
\hline VIII. Vinculación & $\begin{array}{l}\text { C28. Proyección de los beneficios del programa, el } \\
\text { modelo y la institución a empresas y otras instancias } \\
\text { C29. Contribución al conocimiento y trascendencia } \\
\text { del programa mediante colaboraciones } \\
\text { interinstitucionales. }\end{array}$ \\
\hline
\end{tabular}

Fuente: Elaboración propia.
Criterios

C1. El establecimiento de planes y programas de estudio coherente al modelo educativo institucional. $\mathrm{C} 2$. Delimitación de los procesos de evaluación de aprendizajes

C3. Perfil de ingreso y de competencias de egreso establecidos a manera institucional y por programa educativo.

C4. Diseño instruccional y de los objetos de aprendizaje implementados en los programas de Curso.

C5. Establecimiento del proceso de enseñanza y aprendizaje.

C6. Índices de eficiencia terminal a manera institucional y por programa educativo.

C7. Implementación de procedimientos para el seguimiento de egresados.

C8. Desarrollo de investigación y contribución al conocimiento.

C9. Cobertura y trascendencia institucional

c10. Índices de alcance y tendencia de los resultados del modelo

C11. Proceso de admisión y selección establecido.

C12. Procedimientos para el ingreso, permanencia, egreso y titulación del alumno.

C13. Procesos de acompañamiento a la trayectoria escolar como un sistema de tutorias y lo asesorias como programa de apoyo.

C14. Programa de becas o apoyos económicos institucionales.

C15. Núcleo académico.

C16. Evaluación docente y programas de estímulo. C17. Productividad.

C18. Perfil docente enfocado a la educación en modalidad virtual y a distancia.

C19. Equipo, laboratorios y talleres a disposición de docentes y estudiantes.

C20. Campus virtual, plataforma, y todos aquellos elementos vinculados a las TIC. C21. Instalaciones físicas.

$\mathrm{C} 22$. Servicios a los alumnos y funciones del departamento de registro escolar

C23. Bibliotecas digitales, bases de datos, portales de acceso a la información.

C24. Políticas institucionales, reglamento del estudiante, lineamiento escolar, manual de titulación. C25. Compromiso institucional con respecto al aseguramiento de la calidad.

C26. Sistema institucional para el aseguramiento de la calidad.

escolar modelo y la institución a empresas y otras instancias. del programa mediante colaboraciones interinstitucionales. 
La primera dimensión se enfoca al modelo educativo, que comprende criterios asociados a los planes y programas de estudio, los procesos de enseñanza y aprendizaje, el establecimiento de perfiles, y el diseño instruccional. Posteriormente, se encuentran las categorías asociadas a los resultados del modelo, en donde se pretende evaluar la efectividad de los programas educativos con el índice de eficiencia terminal, la cobertura y trascendencia de la institución y del propio modelo.

La tercera dimensión es referida al alumnado, en donde se toman en consideración los procesos de admisión, perfiles de cada estudiante, programas de apoyo al alumno, trayectoria escolar, entre otros. Así mismo, se ha generado una dimensión para la evaluación al personal docente, en la que se categoriza la conformación y resultados de los núcleos académicos, la evaluación al profesor y su productividad.

Enfocada a las herramientas que contribuyen directamente a la formación de los futuros profesionistas se encuentra la dimensión de infraestructura, referida a la evidencia de los equipos, laboratorios, instalaciones, tecnologías, plataformas, bibliotecas, entre otros. De igual forma, se incluyen las funciones de registro escolar, bases de datos, portales, sistemas de información, políticas, reglamentos, etc., mismas que se agrupan en la dimensión de servicios y procesos administrativos.

A través del presente análisis también fue posible observar que los evaluadores se enfocan al compromiso de la institución con el entorno y la calidad misma de las universidades, criterios que pueden ser contenidos en la dimensión de responsabilidad social. Una última dimensión, encaminada a la evaluación de los mecanismos de vinculación, se refiere a los procesos de intercambio y movilidad y la trascendencia en la contribución del conocimiento.

\section{Conclusión}

Mediante los resultados de este análisis teórico y documental puede concluirse que evaluar la calidad educativa no es un proceso fácil o simple, y hasta donde ha sido posible analizar, su evaluación representa un gran reto para los organismos encargados de su acreditación. Se observa que uno de los principales motivos de ello se debe a los constantes cambios e innovaciones tecnológicas, pero estas no han llegado a los sistemas de evaluación; al respecto, se considera que a la par de la innovación y a los cambiantes sistemas educativos, se deberían también desarrollar reestructuraciones en las metodologías de evaluación de la calidad en la educación a distancia.

De la misma manera se hace hincapié en que se deben formular marcos de referencia especializados en la modalidad no convencional, al menos en el contexto mexicano, en donde los sistemas de evaluación fungen principalmente para la evaluación de programas presenciales, que a su vez son utilizados para la evaluación de modalidades no escolarizadas. Así mismo resulta importante encaminar dichos procesos de evaluación a criterios específicos enfocados a dicha modalidad; pues tal y como se ha señalado, la adecuación de los instrumentos utilizados permitirá una evaluación más objetiva, orientada primordialmente a los aprendizajes obtenidos.

También ha sido posible observar que los aspectos evaluados se enfocan más a cuestiones administrativas, infraestructura, recursos disponibles para cada estudiante, entre otros; sin embargo los marcos de referencia carecen de criterios que pretendan evaluar los aprendizajes adquiridos por el alumnado, la aplicación de los conocimientos en el contexto profesional, así como también aquellas percepciones e interpretaciones que este pueda manifestar con respecto al compromiso y satisfacción que le produce el estudiar bajo modalidades no convencionales.

Por ello, se sugiere una reestructuración a los mecanismos de evaluación implementados por las agencias de evaluación, y que estos cambios se encuentren dirigidos a evaluar los aspectos señalados anteriormente, en donde se involucre de mayor manera a cada estudiante, que es verdaderamente en quien se debe medir el impacto educativo. 
Así mismo, consideramos necesario también que los organismos acreditadores unifiquen las terminologías utilizadas en sus sistemas de evaluación, pues existen diferentes denominaciones tales como criterio, estándar, ejes, objetivos, dimensiones, entre otras, las cuales causan confusiones al momento de efectuar los procesos de acreditación y certificación encaminándola a un hecho subjetivo y que se presta mayormente a la interpretación de quien evalúa.

Para finalizar, es preciso enfatizar que precisamos necesario unificar esfuerzos y prestar atención a la evaluación de modalidades educativas no convencionales, particularizando sus criterios dado que, si bien los objetivos entre la modalidad presencial y a distancia son similares, los mecanismos para su ejecución son distintos y sus procesos difieren; lo cual impide que no todos los criterios se cumplan o se logren resultados satisfactorios en las evaluaciones.

\section{REFERENCIAS}

Agencia Nacional de Evaluación de la Calidad y Acreditación. (15 de noviembre de 2018a). ANECA. Recuperado de http://www.aneca.es/ANECA/Presentacion

Agencia Nacional de Evaluación de la Calidad y Acreditación. (15 de noviembre de 2018b). ANECA. Recuperado de http://www.aneca.es/Programas-de-evaluacion

Alcántara, A., (2007). Dimensiones de calidad en la educación superior. Reencuentro,(50), 21-27. Recuperado de htt p://www.redalyc.org/pdf/340/34005004.pdf

Arias, E. (2015). Educación virtual, un reto para la acreditación y el aseguramiento de calidad en Centroamérica. En M. Morocho y C. Rama. (Coord.), Los problemas de la evaluación de la educación a distancia en América Latina yel Caribe (pp. 46-74). Loja: UTPL.

Cervantes, F.; Bañuelos, A.; Chávez, F. y Rocha, J. (2015). La evaluación de la calidad de la educación superior a distancia en México. En Morocho, M. y Rama, C. (Coord.), Los problemas de la evaluación de la educación a distancia en América Latina y el Caribe (pp. 102-130). Loja, Ecuador: Universidad Técnica Particular de Loja.

Comités Insterinstitucionales para la Evaluación de la Educación Superior, A. C. (2017). Programa general para la evaluación de programas educativos de educación superior. Ciudad de México: CIEES.

Consejo Nacional de Ciencia y Tecnología. (2016). Marco de referencia para la evaluación y seguimiento de los programas de posgrados en la modalidad no escolarizada. Ciudad de México: Conacyt.

Diario Oficial de la Federación. (1998). Acuerdo 243. Gobierno de la República. Recuperado de http://www.iea.gob .mx/webiea/inf_general/NORMATIVIDAD

Domínguez, J. (2016). Proceso de acreditación de carreras universitarias en el contexto iberoamericano. En C. Rama y J. Domínguez. (Coord.), El aseguramiento de la calidad de la Educación Virtual (pp. 43-53). Perú: Universidad Católica Los Ángeles de Chimbote.

Fernández, N. (2004). La evaluación y la acreditación de la calidad de la educación superior en América Latina y El Caribe. Buenos Aires: Unesco.

García, L., Ruiz, M., Quintanal, J., García, M., y García, M. (2009). Concepción y tendencias de la educación a distancia en América Latina. Madrid: OEI.

Instituto Latinoamericano y del Caribe de Calidad en Educación Superior a Distancia [CALED]. (2010). Guia de autoevaluación para programas de pregrado a distancia. Loja: CALED

Instituto Latinoamericano y del Caribe de Calidad en Educación Superior a Distancia y Online Learning Consortium [CALED y OLC]. (2015). Tarjeta de puntuación (SCCQAP), evaluación de programas de pregrado en línea. Ecuador: UTPL

Marciniak, R. (2016). Autoevaluación de programas de educación universitaria virtual (tesis doctoral). Universidad Autónoma de Barcelona, Barcelona. 
Marciniak, R. y Gairín J. (2017). Un modelo para la autoevaluación de la calidad de programas de educación universitaria virtual. RED. Revista de Educación a Distancia, (54), 1-30. Recuperado de https://www.redalyc.o $\mathrm{rg} /$ articulo.oa?id=54751771002

Mena, M.; Rodríguez, L. y Diez, M. (2005). El diseño de proyectos de educación a distancia. Páginas en construcción. Buenos Aires: Stella.

Morocho M. y Rama, C. (2015). Los problemas de la evaluación de la educación a distancia en América Latina y el Caribe. Loja: UTPL.

Navarrete, Z y Manzanilla, H. M. (2017). Panorama de la educación a distancia en México. Revista Latinoamericana de Estudios Educativos, 13(1), 65-83. Recuperado de https://bit.ly/39SrpfB

Organización de las Naciones Unidas para la Educación, la Ciencia y la Cultura [Unesco]. (2016). Tecnologías digitales alservicio de la calidad educativa. Una propuesta de cambio centrada en el aprendizajepara todos. Santiago: Unesco.

Rama, C. (2015). Las complejidades de evaluar y acreditar la educación a distancia. En M. Morocho y C. Rama. (Coord.), Los problemas de la evaluación de la educación a distancia en América Latina y el Caribe (pp. 9-16). Loja, Ecuador: Universidad Técnica Particular de Loja.

Rama, C. (2016). Evaluación de la educación no presencial: desde el paradigma tradicional de evaluar procesos de enseñanza (indicadores educativos) a modelos emergentes de evaluación de aprendizajes (competencias adquiridas). En C. Rama y. Domínguez (Ed.), El aseguramiento de la calidad de la Educación Virtual (pp. 25-42). Perú: Universidad Católica Los Ángeles de Chimbote.

Real Academia Española. (10 de abril de 2018). Diccionario de la lengua española. Recuperado de http://dle.rae.es/? $\mathrm{id}=6 \mathrm{nVpk} 8 \mathrm{P} \mid 6 \mathrm{nXVL} 1 \mathrm{Z}$

Rocha, P.; Maina, M. y Sangra, A. (2015). Marco de referencia para la evaluación y aseguramiento de la calidad de los programas de aprendizaje en línea a nivel superior. RED-CUAED. Recuperado de https://bit.ly/2M6zns5

Secretaría de Educación Pública [SEP]. (13 de abril de 2018). SEP, Acciones y programas. Recuperado de https://bit $. l y / 2$ t567LM

CC BY-NC-ND 